\title{
1 Relationships between Influenza viruses A and B and 2 Severe Acute Respiratory Syndrome-Coronavirus-2 3 (SARS-CoV-2) by sequence homologies
}

Makiko Takechi ${ }^{1}$, Shinya Nagasaki ${ }^{2}$, Kibo Nagasaki ${ }^{1,3}$

${ }^{1}$ Tsuchibashi Medical Laboratory, 113-1 Nishikuma Kochi City, Kochi Pref. Japan

82 McMaster University, 1280 Main Street West, Hamilton, Ontario, L8S 4L7, Canada

$9{ }^{3}$ Oakville Christian School, 112 Third Line, Oakville, Ontario, L6L 3Z6, Canada

11 Corresponding Author:

12 1 Makiko Takechi

13 113-1 Nishikuma Kochi City, Kochi Pref. Japan

14 Email address: takechi@tcbs.jp

\section{Abstract}

17 Background: While the severe acute respiratory syndrome coronavirus (SARS-CoV-2) has

18 been spreading and sweeping the world, the number of patients with influenza was much smaller than that in the past years. It is suspected that the coronavirus is not really a new virus that humans experience for the first time. From the aspect of view of gene comparison of influenza A and $\mathrm{B}$ viruses, and coronavirus, they are encoded of identified homologous regions. Methods: The gene sequences of influenza A and B viruses, and coronavirus were identified using data from the National Center for Biotechnology Information (NCBI; Bethesda, MD,

24 USA).

Results: Relatively high homology in amino acid sequences between SARS-CoC-2 and influenza viruses, especially influenza B virus was identified. Coronavirus CDS 9 and CDS 11 encodes proteins that are homologous to proteins of influenza A or B viruses. The results suggest the possibility that antivirals in development or already in use for the medicine treatment of influenza can be effective against coronavirus. This may also suggest the possibility that individuals who experienced an influenza A or B infection within the past 1-2 years would have some immunity to coronavirus. 


\section{Introduction}

37 Japan experienced an influenza epidemic during the 2018-2019 season; in contrast, in 20192020 and 2020-2021, there was a substantial decline in the number of reports of influenza virus infection after the 12th week, 2020 (Figure 1). This finding contrasted with the number of reports of SARS-CoV-2 infection, which increased rapidly during this period. The numbers of influenza patients were 122 and 1316 in March/April 2020 and March/April 2019, respectively. The number of patients who suffered from influenza in 2020-2021 is only 2 as of February 20, 2021. Canada also shows the same tendency

(Figure 2). In many countries, it admitted same tendency. Based on these data, we hypothesized that the coronavirus might be similar to the influenza virus.

Although the SARS-CoV-2 is a novel virus, $80 \%$ of those infected experience mild or asymptomatic disease. As such, we cannot rule out the possibility that the population at large harbors some level of pre-existing immunity due to homology between SARS-CoV-2 and an endemic virus pathogen. Likewise, use of the anti-influenza antiviral agent, Avigan (favipiravir) to coronavirus-infected patients is currently under consideration ${ }^{1}$. Furthermore, some research suggests a possibility of COVID-19 mortality reduction and immunity training by flu vaccination $^{2-5}$.

54 On the other hand, there only a few previous papers that consider potential relationships 55 between gene sequences of influenza viruses and those of SARS-CoV-2. Lai et al. ${ }^{6}$ analyzed fifty-two coronavirus genomes that were available on February 4, 2020 at Global Initiative on Sharing All influenza Data; the authors reported that the reproduction number increased from 0.8 to 2.4 since December 2019. Likewise, Yun et al. ${ }^{7}$ performed a retrospective analysis of nucleic acid sequence and blood test data collected from 2510 virus-infected patients; they reported that the rate of infection with influenza A and B infection was higher than that of SARS-CoV-2.

61 Similar to our study, Chan et al. ${ }^{8}$ characterized coronavirus genomes across species and identified genome-wide patterns of variation encompassing different coronavirus strains; similar variations involving influenza A H1N1 virus were considered. Moreover, Anderson and Reiter ${ }^{9}$

64 reviewed the role of the melatonin-mediated signaling pathway role with respect to the 65 pathogenesis of viral infections, with an emphasis on influenza and COVID-19; their results 
suggested that immune regulators might be appropriate targets in both COVID-19 and other respiratory viral infections. Likewise, Grant et al. ${ }^{10}$ reviewed the roles of vitamin D in reducing the risk of respiratory tract infections. However, to the best of our knowledge, there are no previous studies that examine gene sequence homologies in order to generate predictions regarding both influenza and SARS-CoV-2 infections. As such, in this study, we examined gene sequence homologies of influenza A (A/Puerto Rico/8/1934) and influenza B viruses (B/Lee/1940) with those of SARS-CoV-2 (Wuhan-Hu-1).

Coronavirus is, in general, considered to be a positive-sense single-stranded RNA. However, Taguchi ${ }^{11}$ elucidated that coronavirus has both positive-sense and negative sense characteristics in 2011. CDSs 3 - 12 of SARS CoV-2 have already been segmented in the NCBI database. Therefore, we considered that the function of those segments could be clarified by comparing to influenza virus which is a negative-sense single-stranded RNA.

\section{Materials \& Methods} coronavirus genes of several countries. As illustrated in Table 1, there is a difference of up to $0.03 \%$ with comparison of the Wuhan viral gene to eleven different genes converted to amino acids, only two amino acids are changed. Since these gene are not the location of the start codon or stop codon, it is not important alternation. We also confirmed there are no SARS-CoV-2 compiled of which the difference is more than $0.03 \%$ as of February 13, 2021 in database of the National Center for Biotechnology Information (NCBI; Bethesda, MD, USA). Therefor we decided to analysis using the first Wuhan virus strain discovered.

The function of each genome segment of influenza A and B viruses has been identified. We used this information to guide our comparisons with the SARS-CoV-2 genome. The gene

92 sequences of influenza A virus, influenza B virus and SARS-CoV-2 were identified using data

93 from the NCBI. Given their propensity to undergo mutation, the strains used for each comparison 94 were selected in order to satisfy the following criteria:

95 (1) The genomic sequences of the strains selected must be as close as possible to the original 96 prototype strain. 
97 (2) The function of the specific genomic segments in the influenza viruses required clear and 98 specific documentation.

99 In this study, the following three virus strains were selected for evaluation:

100 1) Influenza $A$ virus, complete genome

101 Influenza A virus (A/Puerto Rico/8/1934(H1N1))

102 RefSeq: GCF_000865725.1

103 2) Influenza $B$ virus, complete genome

104 Influenza B virus (B/Lee/1940)

105 RefSeq: GCF_000820495.2

106 3) SARS-CoV-2, complete genome

107 Wuhan-Hu-1 isolate

108 NCBI Reference Sequence: NC_045512.2

109 The segments' open reading frames encoded by each virus were converted into amino acids 110 used to set protein sequence homology comparisons within the BLAST algorithms in NCBI for 111 the following three virus pairs:

112 (a) Influenza A virus and influenza B virus

113 (b) Influenza A virus and SARS-CoV-2

114 (c) Influenza B virus and SARS-CoV-2

115 This study was conducted in July 2020 and the database and the MCBI Blast algorism 116 available in June 2020 were used.

\section{Results}

119 The values calculated for homology percent as determined by NCBI Blast comparisons of the 120 aforementioned virus sequences are summarized in Tables 2,3 and 4. Table 5 shows the segment 121 function of each of the three viruses, particularly SARS-CoV-2.

As shown in Table 2, many of the sequences encoded by the genome segments of influenza 124 A and influenza B reach 100\% identity, although there is segment with substantially lower 125 sequence homology (0\%-93.75\%). Among these are the PB proteins (PA; PB1; and PB2) that 126 promote virus replication and transcription from virus promoters ${ }^{12,13}$, and others include 
envelope proteins ${ }^{12,13}$ and other surface constituents (NA) ${ }^{12-14}$. When considering all three viruses, significant homologies among individual NP, NA, and M proteins were discovered.

When comparing only the two influenza viruses, segments 4, 5, 6, and segment 7 have $100 \%$ sequence homology; however there are no significant homologies between these segments and similar coding sequences identified in SARS-CoV-2 (Tables 3 and 4). However, the SARS-CoV2 genome coding sequences have high homology $(\geq 80 \%)$, with the same segments encoded by influenza B virus (Table 5); interestingly, CDS 5 and 6 of SARS-CoV-2 have no homology with segment 3 of influenza A. Among the hypotheses, segment 7 of influenza B and CDS 5 of SARS-CoV-2 include sequences encoding the E (envelope) protein, which is an antigen area in SARS-CoV- $2^{15}$. As such, influenza B virus may encode a sequence that has evolved from influenza A.

Our results also suggest that CDS 6 of SARS-CoV-2 sequence corresponding to segment 7 of influenza B virus may encode M1 protein. The CDS 5 and 6 of SARS-CoV-2 may promote functions that are similar to $\mathrm{PA}^{12-14}$ and M1 of influenza B virus ${ }^{14}$. Likewise, CDS 9 of SARSCoV-2 sequence similar to segment 5 of influenza B may function similarly to NP. Sequence homologous to CDS 11 of $\mathrm{N}$ which is one of antigen areas in SARS-CoV-2 ${ }^{16}$ may function similarly to NA protein encoded influenza A and B viruses ${ }^{12-14}$.

The influenza B virus genome encodes an NB protein that is not found within the genome of influenza A virus. Given our hypothesis that influenza B evolved from influenza A virus, this novel protein may have been acquired sometime after the split between these two virus strains. The NB gene encodes a protein with similarities to pathogen recognition receptors ${ }^{14}$, although its precise function with respect to influenza $B$ virus infection remains unclear. Likewise, the functions of influenza A proteins PA and M2 remain undefined; as such, it is perhaps not surprising to find no specific homology between sequences encoding these two influenza A proteins and genome sequence of SARS-CoV-2. With respect to development of novel antiviral medicines and/or vaccines, researchers might focus on the most highly homologous segments so 


\section{Discussion}

159 Many of the most significant differences between influenza A virus, influenza B virus and 160 SARS-CoV-2 relate to the distinct sequences encoding the $\mathrm{M}^{12,13}$ and the $\mathrm{NP}^{12-14}$ proteins. In 161 this study, we identified sequence homologies among proteins encoded by the influenza A virus, 162 influenza B virus, and SARS-CoV-2; putative functions for four of the 12 CDSs of the SARS$163 \mathrm{CoV}-2$ genome were revealed. Furthermore, the relative high homology among these sequences 164 may suggest the possibility that individuals who experienced infections with either influenza A 165 or influenza B within 1-2 years might have mild or no symptoms of COVID-19. An

166 epidemiological survey directed specifically at this hypothesis might be undertaken in the near 167 future. results of this study suggest that one or more of the antivirals in use or in development to combat influenza infection might be examined for use against SARS-CoV-2 infection. Coronavirus is

\section{Conclusions}

The genome of the coronavirus pathogen, SARS-CoV-2, includes four regions that display high homology ( $80 \%$ or more) with genome segments that are unique to influenza B virus. As such, this study may be among the first to consider the possibility that antivirals directed against influenza might be effective for the treatment COVID-19. Notably, this homology involves CDSs 5, 6, 9, and 11 of SARS-CoV-2. Currently, SARS-CoV-2 is fractionated into twelve distinct CDSs; ongoing review of these designations will be necessary as progress in this field 185 verification.

\section{Acknowledgements}

188 The authors would like to thank Enago (www.enago.jp) for the English language review. 


\section{References}

1. Alexander SPH, Armstrong J, Davenport AP, et al. A rational roadmap for SARS-CoV2/COVID-19 pharmacotherapeutic research and development: IUPHAR Review 29. Br J Pharmacol 2020; https://doi.org/10.1111/bph.15094.

2. Zanettini C, Omar M, Dinalankara W, et al. Influenza vaccination and COVID-19 mortality in the USA: medRxiv 2020; https://doi.org/10.1101/2020.06.24.20129817.

3. Fink G, Orlova-Fink N, Schindler T, Grisi S, et al. Inactivated trivalent influenza vaccine is associated with lower mortality among COVID-19 patients in Brazil: medRxiv 2020; https://doi.org/10.1101/2020.06.29.20142505.

4. Noale M, Trevisan C, Maggi S, et al. The association between influenza and pneumococcal vaccinations and SRAS-CoV-2 infection: Data from the EPICOVID19 web-based survey: Vaccines 2020; 8(3): 471; https://doi.org/10.3390/vaccines8030471.

5. Debisarun PA, Struycken P, Domínguez-Andrés J, et al. The effect of influenza vaccination on trained immunity: impact on COVID-19: medRxiv 2020; https://doi.org/10.1101/2020.10.14.20212498.

6. Lai A, Bergna A, Acciarri C, et al. Early phylogenetic estimate of the effective reproduction number of SARS-CoV-2. J Med Viol 2020; 92: 675-679.

7. Yun H, Sun Z, Wu J, et al. Laboratory data analysis of novel coronavirus (covid -19) screening in 2510 patients. Clin Chim Acta 2020; 507: 94-97: https://doi.org/10.1016/j.cca.2020.04.018.

213 9. Anderson G and Reiter RJ. Melatonin: Roles in influenza, Covid-19, and other viral 214 infections. Rev Med Virol 2020; 30: e2109: https://doi.org/10.1002/rmv.2109.

215 10. Grant WB, Lahore H, McDonnell SL, et al. Evidence that vitamin D supplementation could 216 reduce risk of influenza and COVID-19 infections and death. Nutrients 2020;12:988-1006: 217 https://doi.org/10.3390.2020.

218 11. Taguchi F, Coronaviruses, Virus 2011; 61(2): 205-210 (in Japanese). 
219 12. Gao Q, Chou Y-Y, Doganay S, et al. The Influenza A virus PB2, PA, NP, and M segments 220 play a pivotal role during genome packaging. J Virol 2012; 86: 7043-7051:

221 https://doi.org/10.1128/JVI.00662-12.

222 13. Kim JI, Lee I, Park S, et al. Reassortment compatibility between PB1, PB2, and HA genes 223 of the two influenza B virus lineages in mammalian cells. Sci Rep 2016; 6: 27480 :

224 https://doi.org/ 10.1038/srep27480.

225 14. Krammer F, Smith GJD, Fouchier RAM, et al. Influenza. Nat Rev Dis Primers 2018; 4: 3: 226 https://doi.org/10.1038/s41572-018-0002-y.

227 15. DeDiego ML, Álvarez E, Almazán F, et al. A severe acute respiratory syndrome coronavirus 228 that lacks the $\mathrm{E}$ gene is attenuated in vitro and in vivo. $J$ Virol 2007; 81: 1701-1713: 229 https://doi.org/10.1128/JVI.01467-06.

230 16. Zhao X, Nicholls JM and Chen,YG. Severe acute respiratory syndrome-associated 231 coronavirus nucleocapsid protein interacts with Smad3 and modulates transforming growth 232 factor-beta signaling. J Biol Chem 2008; 283: 3272-3280:

233 https://doi.org/10.1074/jbc.M708033200. 
236 Table 1

237 Comparison of Coronavirus-2 (SARS-CoV-2) among Wuhan coronavirus

238 (NC_045512.2) and other coronaviruses identified in some countries analyzed with 239 NCBI blast.

241 Table 2

242 Homology (\%) between proteins encoded by segments of the RNA genomes of 243 influenza A H1N1 (A/Puerto Rico/8/1934) and influenza B virus (B/Lee/1940); S, 244 segment number.

\section{Table 3}

247 Homology (\%) between proteins encoded by segments of the RNA genome of influenza 248 A H1N1 (A/Puerto Rico/8/1934) and proteins encoded by the genome of the SARS-

249 CoV-2 Wuhan-Hu-1 isolate. The function of each genome segment and coding 250 sequence (CDS) are as shown.

252 Table 4

253 Homology (\%) between proteins encoded by segments of the RNA genome influenza B 254 virus (B/Lee/1940) and proteins encoded by the genome of the SARS-CoV-2 Wuhan$255 \mathrm{Hu}-1$ isolate. The function of each genome segment and coding sequence (CDS) are as 256 shown.

\section{Table 5}

Combinations of segments and encoded proteins that display high homology ( $\geq 80 \%)$ between influenza A H1N1 (A/Puerto Rico/8/1934) or influenza B virus (B/Lee/1940)

262 represent homologies between influenza A H1N1 and SARS-CoV-2 / homologies

263 between influenza B and SARS-CoV-2. The areas where the three viruses have 264 common proteins are shown in gray. 
Figure 1

276 Seasonal variation in the numbers of positive tests for influenza virus and the number of 277 positive PCR tests for SARS-CoV-2 in Japan. The pink, red, green and black lines 278 represent the number of positive tests for influenza in 2020-2021, 2019-2020, 20182792019 and 2017-2018, respectively. The solid and dotted lines represent total number of 280 positive tests for influenza A and B, and influenza A only, respectively. The blue line 281 represents the number of positive PCR tests for SARS-CoV-2. Report week 1 282 corresponds to January 4 to January 10, 2021 in 2020-2021, December 30, 2019 to 283 January 5, 2020 in 2019-2020, December 31, 2018 to January 6, 2019 in 2018-2019, 284 and January 1, 2018 to January 7, 2018 in 2017-2018. The number of patients is 285 opened by national institute of infectious diseases.

\section{Figure 2}

289 Seasonal variation in the numbers of positive tests for influenza virus and the number of 290 positive PCR tests for SARS-CoV-2 in Canada. The pink, red, green and black lines 291 represent the number of positive tests for influenza in 2020-2021, 2019-2020, 20182922019 and 2017-2018, respectively. The solid and dotted lines represent total number of 293 positive tests for influenza A and B, and influenza A only, respectively. The blue line 
294 represents the number of positive PCR tests for SARS-CoV-2. Report week 1

295 corresponds to January 4 to January 10, 2021 in 2020-2021, December 30, 2019 to

296 January 5, 2020 in 2019-2020, December 31, 2018 to January 6, 2019 in 2018-2019,

297 and January 1, 2018 to January 7, 2018 in 2017-2018. The number of patients is

298 opened by Government of Canada.

299 